\title{
Editorial
}

\section{Online search charges - Evolution, devolution, transition}

The three traditional operators in the online marketplace: users, database producers, and hosts are in the throes of making adjustments to environmental pressures for change. For almost 15 years, the charging modalities have, if nothing else, been reliable and standardized.

Now we are beginning to see this vital component wriggle about with a variety of mutations emerging. As might be expected, these mutations will not all have survival potential, and they are by definition, not standardized.

Readers of ISU, no matter which role they play, are probably mystified, enraged, or experiencing a variety of emotions as they confront these evolutions.

Emotional reactions will not lead to constructive interplay, yet as 'pocket-book' issues, the charges paid for the use of systems and databases generate sparks from even the most placid.

At this time I think it appropriate to put aside emotions and look at the facts.

1. It is a fact that users have increasing difficulty in commanding the requisite funding to support the use of increasingly expensive online services. While the most expert searchers use every intellectual trick and exploit every technological advantage to keep costs down, even they are fighting a losing battle. Efficiency increases have finite limits and price increases seem to have none. The less experts are in even worse economic shape.

2. It is a fact that database manufacturing is becoming more and more expensive. The available buffer that many producers used to subsidize online files (profits from printed counterparts) is shrinking rapidly. Migration - the shift in use from print to online - creates a negative revenue effect. Coupled with increasing manufacturing costs, the only available counterattack seems to lie in either increasing prices for the charging modalities or to find new ones possibly both.

3. It is a fact that hosts are caught squarely in the middle of these pressures. Their options for efficiency enhancements are technologically bound. Unless they can make breakthroughs with software and hardware combinations, they 
can have little effect on the situation. While they have some control in the software area, there is little that they can do to provide a hardware breakthrough - that must come from others.

An examination of the elements of the old charging modalities, reveals their dependence on basic technological elements. These in turn lead to their expression in the marketplace. The concept of 'time-sharing' based on central processing unit (CPU) costs and data storage factors produced a time-centered structure. Accounting systems installed for time sharing provided the 'connect hour' as the basic charging modality. We can ascribe the focus on this method primarily to the host organizations - it was the common element in the online searching process. The introduction of 'hit' (output) charges on the other hand, had its origins with the database producers who saw this component as a reasonable follow on to the active search process. Many producers delayed the introduction of this component for a time, but eventually 'hit' charging became the standard.

There have been some minor user inputs to the charging structure. The adoption of 'free' formats has been largely in response to user rationales. While producers accepted these rationales, there has been a trend in recent years to reduce their liberality.

With this minor exception, the time/output standard has served us (if not well) reliably for many years.

Technological advances, e.g., increased baud rates, new system capabilities, and time-independent media (CD-ROM) have strained the traditional method and in recent years new approaches have been advocated and installed.

Possibly the most dramatic (and emotional) change came from Chemical Abstracts Service (CAS), but hosts have introduced new approaches (sometime under the guise of system enhancements) eroding confidence and introducing anxieties.

In our opinion the period of change we are experiencing is natural, evolutional, and inevitable. The harmonization of new elements requires experiment and experience. Flaws in communications, old prejudices, will be resolved, must be resolved to a vital system whose values far exceed these temporary perturbations.

A.W. Elias 